\title{
Erratum: Carotenoids and Fatty Acids in Red Yeasts Sporobolomyces roseus and Rhodotorula glutinis" [Applied Biochemistry and Microbiology, vol. 40, no. 4, 2004, p. 392]
}

\section{P. Davoli, V. Mierau, and R. W. S. Weber}

Below is the correct version of Tables 1-3 and the caption to Fig. 2.

Table 1. Quantification of carotenoids ( $\mu$ g carotenoids $\mathrm{g}^{-1}$ dry weight) in shaken flask cultures of $R$. glutinis and S. roseus. The factor by which carotenoid production increased in indented relative to straight flasks is also given

\begin{tabular}{|c|c|c|c|c|c|c|}
\hline \multirow{3}{*}{ Pigments } & \multicolumn{3}{|c|}{ Rhodotorula glutinis } & \multicolumn{3}{|c|}{ Sporobolomyces roseus } \\
\hline & \multicolumn{3}{|c|}{ flask type } & \multicolumn{3}{|c|}{ flask type } \\
\hline & straight & indented & factor & straight & indented & factor \\
\hline$\beta$-Carotene & $44.3 \pm 6.5$ & $74 \pm 19$ & 1.7 & $72 \pm 12$ & $101 \pm 30$ & 1.4 \\
\hline$\gamma$-Carotene & $15.2 \pm 1.9$ & $19.2 \pm 4.5$ & 1.3 & $11.8 \pm 3.7$ & $67 \pm 16$ & 5.7 \\
\hline Torulene & $51.5 \pm 7.1$ & $107 \pm 46$ & 2.1 & $20.8 \pm 4.3$ & $203 \pm 50$ & 9.8 \\
\hline Torularhodin & $2.13 \pm 0.28$ & $6.0 \pm 2.3$ & 2.8 & $4.1 \pm 1.6$ & $41.2 \pm 8.8$ & 10.2 \\
\hline
\end{tabular}

Table 2. Fatty acid composition (\% of total) of R. glutinis and S. roseus grown in straight and shaken flasks. All fatty acids were determined by GC-MS as their methyl esters

\begin{tabular}{l|c|c|c|c}
\hline \multirow{2}{*}{\multicolumn{1}{c|}{ Fatty acids }} & \multicolumn{2}{c|}{ Rhodotorula glutinis } & \multicolumn{2}{c}{ Sporobolomyces roseus } \\
\cline { 2 - 5 } & \multicolumn{2}{|c}{ flask type } & \multicolumn{2}{c}{ flask type } \\
\cline { 2 - 5 } & straight & indented & straight & indented \\
\hline $\mathrm{C}_{14: 0}$ tetradecanoic acid & $1.21 \pm 0.45$ & $1.53 \pm 0.45$ & $1.12 \pm 0.19$ & $2.13 \pm 0.17$ \\
$\mathrm{C}_{15: 0}$ pentadecanoic acid & $0.52 \pm 0.14$ & $0.38 \pm 0.04$ & $0.39 \pm 0.19$ & $0.33 \pm 0.08$ \\
$\mathrm{C}_{16: 1}$ (Z)-9-hexadecenoic acid & $2.08 \pm 0.53$ & $1.23 \pm 0.22$ & $1.75 \pm 0.06$ & $3.93 \pm 0.46$ \\
$\mathrm{C}_{16: 0}$ hexadecanoic acid & $15.52 \pm 0.77$ & $20.0 \pm 1.9$ & $13.38 \pm 0.11$ & $20.4 \pm 1.8$ \\
$\mathrm{C}_{17: 1}$ 15-methyl-11-hexadecanoic acid & $1.28 \pm 0.26$ & $0.36 \pm 0.03$ & $0.56 \pm 0.07$ & $1.16 \pm 0.05$ \\
$\mathrm{C}_{17: 0}$ heptadecanoic acid & $0.38 \pm 0.06$ & $0.27 \pm 0.03$ & $0.59 \pm 0.04$ & $0.28 \pm 0.02$ \\
$\mathrm{C}_{18: 2}$ (Z,Z)-9.12-octadecadienoic acid & $72.1 \pm 1.6$ & $69.1 \pm 2.3$ & $60.2 \pm 2.1$ & $64.1 \pm 1.9$ \\
$\mathrm{C}_{18: 1}$ (Z)-9-octadecenoic acid & $5.72 \pm 0.52$ & $6.07 \pm 0.80$ & $16.58 \pm 0.71$ & $5.45 \pm 0.74$ \\
$\mathrm{C}_{18: 0}$ octadecanoic acid & $0.45 \pm 0.19$ & $0.19 \pm 0.16$ & $1.47 \pm 0.42$ & $0.70 \pm 0.35$ \\
$\mathrm{C}_{20: 1}$ 11-eicosenoic acid & $0.11 \pm 0.05$ & $0.09 \pm 0.08$ & $1.05 \pm 0.26$ & $0.31 \pm 0.21$ \\
$\mathrm{C}_{20: 0}$ eicosanoic acid & $0.36 \pm 0.17$ & $0.55 \pm 0.29$ & $1.55 \pm 0.48$ & $0.64 \pm 0.36$ \\
$\mathrm{C}_{22: 0}$ docosanoic acid & $0.24 \pm 0.16$ & $0.26 \pm 0.02$ & $1.39 \pm 1.10$ & $0.53 \pm 0.53$ \\
$\mathrm{C}_{24: 0}$ tetracosanoic acid & \multicolumn{3}{c}{} \\
\hline
\end{tabular}

Table 3. Total carotenoid and fatty acid balances for $R$. glutinis and S. roseus grown in straight and indented shaken flasks

\begin{tabular}{c|c|c|c|c}
\hline \multirow{2}{*}{ Parameter } & \multicolumn{2}{|c|}{ Rhodotorula glutinis } & \multicolumn{2}{c}{ Sporobolomyces roseus } \\
\cline { 2 - 5 } & \multicolumn{2}{|c|}{ flask type } & stask type \\
\cline { 2 - 4 } & straight & indented & $109 \pm 21$ & $412 \pm 103$ \\
\hline Total carotenoids & $113 \pm 14$ & $206 \pm 72$ & $203 \pm 40$ & $766 \pm 192$ \\
$\mu \mathrm{g} \mathrm{g}^{-1}$ dry weight & $211 \pm 27$ & $385 \pm 134$ & & \\
$\mathrm{nmol} \mathrm{g}^{-1}$ dry weight & $19.7 \pm 6.2$ & $19.5 \pm 7.1$ & $14.3 \pm 4.9$ & $42 \pm 23$ \\
Total fatty acids (FAs) & $70 \pm 21$ & $70 \pm 21$ & $51 \pm 14$ & $160 \pm 68$ \\
$\mathrm{mg} \mathrm{g}^{-1}$ dry weight & & & & \\
$\mu$ mol g-1 dry weight & $0.33 \pm 0.14$ & $0.55 \pm 0.04$ & $0.42 \pm 0.15$ & $0.50 \pm 0.11$ \\
Carotenoids/FAs & & \\
mol-percent &
\end{tabular}

Fig. 2 . The carotenoid composition in response to changes in culture conditions. The left-hand columns of each pair show extracts from straight flask cultures, right-hand columns show indented flasks. (1) $\beta$-carotene, (2) $\gamma$-carotene, (3) torulene, (4) torularhodin. 